\title{
Hintergrund: Die „Große Transformation" im Kontext von Forschung und Politik
}

Im folgenden Kapitel wird zunächst der Hintergrund erläutert, vor dem die transformative Forschung als Ansatz entwickelt wurde: Die Umweltfolgen menschlichen Handelns im Zeitalter des Anthropozän, die damit zusammenhängende Kritik an einer auf dauerhaftem Wachstum basierenden Gesellschaft sowie die Schlussfolgerungen zur Notwendigkeit einer Transformation (Abschn. 2.1). Damit zusammenhängend entstanden verschiedene Forschungsansätze, welche an eine größere wissenschaftstheoretische und wissenschaftspolitische Debatte über eine Neuorientierung der Wissenschaft angesichts globaler Herausforderungen anschließen. Während die Transformationsforschung die geforderten und stellenweise bereits gelungenen Veränderungsprozesse lediglich $\mathrm{zu}$ verstehen versucht, intendieren Transition-Forschung und transformative Forschung, mögliche Visionen zu entwickeln und den Prozess mit zu begleiten (WBGU 2011, S. 23). Diese sowie verwandte Forschungsperspektiven im Kontext der Forderung nach einer Transformation werden in Abschnitt 2.2 dargestellt. Daraufhin werden Städte in diesen Kontext eingeordnet, mit ihren Handlungsspielräumen und Möglichkeiten, lokal Transformationen anzustoßen (Abschn. 2.3) sowie der spezifische institutionelle Kontext Wuppertals beleuchtet (Abschn. 2.4). Den Abschluss des Hintergrundkapitels bildet ein Zwischenfazit, das dazu dient, die relevanten Annahmen der geschilderten Forschungsperspektiven für die Analyse aufzubereiten (Abschn. 2.5).

\subsection{Nachhaltige Entwicklung, Grenzen des Wachstums und die „Große Transformation“}

Das aktuelle Zeitalter des Anthropozän, in dem sich die Erde laut Ergebnissen aus Klima- und Umweltforschung seit Mitte des 18. Jahrhunderts befindet, ist 
dadurch gekennzeichnet, dass die Menschheit durch ihre Aktivitäten zum ersten Mal das Erdsystem beeinflusst (Crutzen 2002). Insbesondere in den vergangenen Jahren sind dabei verstärkt Prozesse zu beobachten, durch die mit einer hohen Wahrscheinlichkeit irreversible Umweltveränderungen eintreten könnten, die das Erdsystem als solches verändern und dessen Folgen schwer abzuschätzen sind. Rockström et al. (2009; sowie Steffen et al. 2015) haben in ihrem Konzept planetarer Leitplanken neun Prozesse definiert, die zentral für die Funktion und Stabilität des Erdsystems sind. Zwei werden besonders herausgestellt: Der Klimawandel und das Funktionieren von Ökosystemen beziehungsweise der Verlust von Biodiversität, da sie alleine das Erdsystem aus dem Gleichgewicht bringen könnten. Bei allen diesen Prozessen gibt es Kipppunkte, ab deren Überschreiten voraussichtlich irreversible Schäden entstehen und sich Effekte verstärken, wodurch es zu rapiden, plötzlichen Veränderungen kommen kann. Bereits kurz vor Erreichen dieser Schwellen setzen die Autoren die sogenannten planetaren Leitplanken - Zeitpunkte, ab denen große Unsicherheiten über die Entwicklung des Erdsystems bestehen, die also als Warnung gelten sollten, um Prozesse umzukehren. Im Fall der Ozonschicht, so schreiben sie, wurde eine planetare Leitplanke bereits überschritten. Die Entwicklung konnte durch politische Anstrengungen jedoch wieder umgekehrt werden, so dass die Ozonschicht sich größtenteils regeneriert hat. Die Autoren gehen davon aus, dass mehrere andere Leitplanken bereits überschritten sind: der Klimawandel, der Biodiversitätsverlust, die Stickstoff- und Phosphorkreisläufe und die Landnutzung (Rockström et al. 2009; Steffen et al. 2015). Die Autoren sind sich sicher bezüglich des Einflusses der Menschheit auf die Prozesse im Erdsystem, andere Fragen bleiben jedoch bislang offen. So ist bisher an vielen Stellen unklar, wie genau die Prozesse miteinander zusammenhängen und sich gegenseitig verstärken (Steffen et al. 2015, S. 7).

Das Konzept der planetaren Leitplanken ist eines der bekannten Konzepte hinsichtlich der Umweltauswirkungen der Menschheit, doch bei weitem nicht das einzige. Auch in anderen Publikationen wird vor irreversiblen Umweltveränderungen gewarnt und diese Warnung in vielen Fällen auch an die Politik herangetragen. Besonders prominent geschieht dies in den regelmäßigen Berichten des Intergovernmental Panel on Climate Change (IPCC, dt. Weltklimarat), von denen bereits 1992 der erste erschien (IPCC 1992) oder in den Veröffentlichungen des Intergovernmental Science-Policy Platform on Biodiversity and Ecosystem Services (IPBES, dt. Weltbiodiversitätsrat), beispielsweise 2019 mit einer Auswertung zahlreicher Studien zum Artensterben (IPBES 2019).

Diese und andere Berichte treffen die klare Aussage, dass Veränderungen notwendig sind, um die Ressourcennutzung, den Schadstoffausstoß und Landnutzungen zu verändern. In diesem Zusammenhang kam auch das Ziel nachhaltiger 
Entwicklung auf, das die Weltkommission für Umwelt und Entwicklung (WCED, auch Brundtlandkommission) bereits in ihrem Bericht 1987 formulierte. Hier wird nachhaltige Entwicklung definiert als ,,to ensure that it meets the needs of the present without compromising the ability of future generations to meet their needs" (WCED 1987). Später einigten sich die Mitgliedstaaten der Vereinten Nationen (UN) und ihrer Unterorganisationen in weiteren Abkommen auf das Ziel einer nachhaltigen Entwicklung, so in der Rio-Deklaration (UNCED 1992), der Agenda 21 (UN DSD 1992), dem Paris-Abkommen (UN 2015b) oder in der Agenda 2030 (UN 2015a).

Neben diesen internationalen Prozessen wird das Thema Nachhaltigkeit auch auf nationaler und lokaler Ebene adressiert. Hintergrund dessen ist unter anderem, dass in Städten Umweltfolgen schnell sichtbar werden, aber auch, dass mittlerweile mehr als die Hälfte der Menschheit in Städten wohnt. Dadurch entsteht ein großer Teil des Ressourcenverbrauchs in Städten und eine Entwicklung hin zu nachhaltigem urbanen Leben erscheint wichtiger denn je (UN-Habitat 2016; WBGU 2016).

Grundlage der Idee nachhaltiger Entwicklung als wirtschaftliche Entwicklung mit geringerem Umweltverbrauch ist es, dass durch eine Entkopplung bei gleichbleibender Wirtschaftsaktivität durch den Einsatz effizienterer Technologien weniger Ressourcen verbraucht werden (Rees 1995; Robinson und Tinker 1998). Doch in der Praxis zeigt sich, dass sogenannte Rebound-Effekte dafür sorgen, dass bei gesteigerter Ressourceneffizienz nicht die theoretisch errechnete Einsparung erreicht wird, sondern durch Konsumverschiebung und -steigerung der Verbrauch insgesamt direkt oder indirekt die Einsparungen verringert oder diese gar ausbleiben (Sorell 2009). Deshalb argumentieren Kritiker der klassischen Argumentation nachhaltiger Entwicklung, dass zusätzlich auch eine Entkopplung der Lebensqualität von der Wirtschaftsaktivität gelingen muss (u. a. Raskin et al. 2002; Robinson und Tinker 1998; Schneidewind 2018, S. 65). Diese absolute Entkopplung, also eine wirkliche Verringerung des Ressourcenverbrauchs bei gleichbleibender oder erhöhter Lebensqualität, wurde jedoch bisher kaum oder gar nicht erreicht (Jackson 2009, S. 67-73; Wegner 2013, S. 9 f.). Jackson (2009, S. 118) argumentiert,

„There is still no consistent vision of an economy founded on continual consumption growth that delivers absolute decoupling. And the systemic drivers of growth push us relentlessly towards ever more unsustainable resource throughput“.

Damit zusammenhängend wird schon seit langer Zeit Kritik daran geäußert, dass oftmals davon ausgegangen wird, nachhaltige Entwicklung solle stets mit 
Wirtschaftswachstum einhergehen (u. a. Robinson und Tinker 1998, S. 21). Dies bedeutet, dass Effizienzsteigerungen alleine nicht helfen, sondern dass auch Veränderungen in Politik und Konsummustern, in Lebensstilen und Werten notwendig sind (BUND und Misereor 1997, S. 101-113; Robinson und Tinker 1998, S. 33-35; Wegner 2013, S. 9 f.). Hieran schließen auch die Argumente derjenigen an, die mit verschiedenen Konzepten wie Postwachstum oder Suffizienz das ständige Wirtschaftswachstum hinterfragen und alternative Wege aufzeigen (u. a. Jackson 2009; I. Seidl und Zahrnt 2013).

Bereits in den 1970er Jahren argumentierte der Club of Rome (Meadows et al. 1972), dass dauerhaftes Wachstum in einer begrenzten Welt nicht möglich sei und andere Indikatoren für Lebensqualität notwendig seien. Vor diesem Hintergrund argumentieren viele der Befürworter*innen erweiterter Wohlstandsmodelle. Sie argumentieren, dass, solange Wohlstand nur an materiellen Faktoren und damit vor allem Konsum gemessen wird, absolute Entkopplung kaum möglich ist. Ein erweitertes Wohlstandsverständnis dagegen würde eine hohe Lebensqualität aller Bevölkerungsgruppen unabhängig von einem erhöhten Ressourcenverbrauch ermöglichen (u. a. Zahrnt und Schneidewind 2015, S. 73). Notwendig sei, so Zahrnt und Schneidewind (2015, S. 73), eine Suffizienzpolitik, die auf neuen Wohlstandsmaßen basiert, welche auch immaterielle Aspekte abbilden können. Eine Veränderung der vorherrschenden Paradigmen, insbesondere des Wachstumsparadigmas, ist also als große aber notwendige Aufgabe zu sehen (Göpel 2016, S. 3; Sievers-Glotzbach und Tschersich 2019, S. 6 f.).

Daher beschäftigen sich zahlreiche Wissenschaftler*innen, Politiker*innen und andere Akteur*innen mit der Suche nach diesem neuen „Kompass“ und damit nach einer geeigneten Definition und zugehörigen Indikatoren für Lebensqualität. Sie äußern Kritik am Bruttoinlandsprodukt (BIP) als viel verwendetem Indikator für eben diese Lebensqualität und schließen sich damit einer schon seit den 1960er Jahren laufenden Diskussion an (Delhey 2013, S. 147 f.; Stiglitz et al. 2009).

Ein grundsätzlicher Kritikpunkt am BIP ist, dass es nur Dinge und Prozesse mit monetärem Wert beinhaltet. Verschiedenste Aspekte des guten Lebens liegen allerdings außerhalb der Märkte, sind nicht monetär messbar und fließen daher nicht in das BIP ein, haben aber dennoch einen Einfluss auf die Wirtschaft und Gesellschaft - wie Haushaltsarbeit und Pflege. Außerdem gibt es negative Kosten, die, wenn sie monetär messbar sind, positiv in das BIP eingerechnet werden, auch wenn sie einen negativen Effekt auf die Lebensqualität haben, wie Krankheitsoder Umweltkosten (BUND und Misereor 1997, S. 96 f.; Jackson 2009, S. 125).

Insbesondere seit den 1970er Jahren wurden zahlreiche Umwelt- und Ressourcenindikatoren entwickelt. Später wurden immer häufiger Indikatoren für 
Nachhaltigkeit sowie für Lebensqualität entwickelt und in verschiedenen Politikbereichen eingeführt (Lehtonen 2015, S. 77). Einige werden unter dem Begriff Nachhaltigkeitsindikatoren gefasst (u. a. IAEG-SDG 2016; Landesregierung Nordrhein-Westfalen 2016; Science for Environment Policy und UWE 2018; Statistisches Bundesamt 2016), andere als Wohlstandsindikatoren bezeichnet (u. a. Canadian Index of Wellbeing 2016; OECD 2011). Sie wurden sowohl für die Ebene von Staaten als auch für einzelne Regionen und Städte entwickelt $^{1}$. Darüber hinaus werden ähnliche Indikatoren oftmals in Städterankings verwendet, die Städte im Hinblick auf verschiedene Aspekte wie Wirtschaft und Lebensqualität vergleichen (z. B. Städteranking der Wirtschaftswoche: Losse und Fischer 2015; HWWI/Berenberg-Städteranking: Nitt-Drießelmann und Wedemeiner 2015) sowie in Bezug auf Nachhaltigkeitsaspekte (Morgenstadt City Index: von Radecki et al. 2016). Einige Indikatorensets hinterfragen dabei explizit das Wachstumsparadigma, während andere eher als Ergänzung zum BIP entwickelt werden und Wirtschaftswachstum nicht in Frage stellen.

Seit der Rio-Konferenz 1992 wurden mit dem Brundtlandbericht und der Agenda 21 Indikatoren von den meisten Regierungen weltweit als wichtig deklariert, um eine Entwicklung hin zu Nachhaltigkeit zu fördern und zu evaluieren (Rametsteiner et al. 2011, S. 61 f.). Dies zeigt auch die Entwicklung des Better Life Index (BLI) durch die Organisation für Wirtschaftliche Zusammenarbeit und Entwicklung (OECD), die seit 2011 alle zwei Jahre das gute Leben in den OECD-Staaten in elf Dimensionen und mit dazugehörigen Indikatoren abbildet.

Die Einrichtung der Enquete-Kommission des Deutschen Bundestages „Wachstum, Wohlstand, Lebensqualität“, die von 2011 bis 2013 Möglichkeiten der Messung von Lebensqualität diskutierte (Deutscher Bundestag 2013) sowie der 2016 erschienene Bericht der Bundesregierung „Gut Leben in Deutschland“ (Presse- und Informationsamt der Bundesregierung 2016) zeigen, dass auch in Deutschland in vielen Bereichen der Politik weiterhin nach ergänzenden oder alternativen Indikatoren gesucht wird, an denen politische Entscheidungen ausgerichtet und Entwicklungen gemessen werden können. So kam die EnqueteKommission zu der gemeinsamen Aussage, dass das BIP kein geeigneter Indikator ist, um Entwicklungen und Wohlstandsniveau zu messen (Deutscher Bundestag 2013, S. 23).

Diese Prozesse in und außerhalb Deutschlands lassen darauf schließen, dass die BIP-Kritik beziehungsweise die Forderung nach einer Erweiterung dieses

\footnotetext{
${ }^{1}$ Für einen Vergleich der Indikatorensysteme siehe u. a. OECD (2013, 2014, 2015), Schepelmann et al. (2010), Widuto (2016), EC und Statistical Office of the European Communities (2005), Science for Environment Policy und UWE (2018).
} 
Indikators in Politik und Wissenschaft vermehrt an Bedeutung gewonnen hat (Hayden und Wilson 2017, S. 170). Laut einer Studie von Hayden und Wilson (2017, S. 181) liegt ein möglicher Grund dafür, dass alternative Indikatoren sich bisher schwer etablieren ließen und eine längerfristige Nutzung dieser Indikatoren bisher nicht gelungen ist, in der schwierigen Kommunikation der Konzepte „Glück“, „Wohlstand“ und „Wohlbefinden“, da diese Themen in Gesellschaft und Politik oft nicht ernst genommen würde.

Vor diesem Hintergrund wurde in den vergangenen Jahren die Forderung nach einer Transformation in Richtung Nachhaltigkeit lauter (u. a. IPCC 2012; WBGU 2011). Um das Leben der Menschen innerhalb planetarer Leitplanken langfristig zu ermöglichen, ist ein grundlegender Wandel notwendig, der über kleine Veränderungen wie Effizienzsteigerungen oder Anpassungen innerhalb bestehender Institutionen und Systeme hinausgeht. Dieser Wandel beinhaltet eine Veränderung in Wirtschaft, Politik und Gesellschaft (Kemp und van Lente 2013, S. 116; Loorbach 2017, S. 193; WBGU 2011). Der WBGU (2011, S. 1 f.) verwendet für diesen Prozess den Begriff „Große Transformation" und bezieht sich dabei auf Polanyi (1944), der mit der „Great Transformation“ den Umbruch zur industrialisierten Wirtschaft und Gesellschaft bezeichnete. Im Kontext der Nachhaltigkeitswissenschaft meint „Große Transformation“ folglich eine grundlegende Umgestaltung der Gesellschaft hin zu einer umweltverträglichen, nachhaltigen Lebensweise. So wird eine Transformation oder Transition als ,far-reaching change“ (Brand 2016, S. 24) beziehungsweise als „broad social, ecological and economic changes“ (Martens und Rotmans 2005, S. 1136) bezeichnet. Dabei handelt es sich um Veränderungen der bestehenden Wirtschafts-, Politik- und Gesellschaftssysteme und nicht lediglich einzelner Bereiche (Martens und Rotmans 2005, S. 1136; WBGU 2011, S. 89 f.).

Im Gegensatz zu einigen anderen Transformationen hat die Nachhaltigkeitstransformation ein definiertes Ziel - Veränderungen hin zu einer nachhaltigen Gesellschaft (Geels 2011, S. 25; Nevens et al. 2013, S. 112). Diese Zielvision ist laut WBGU (2011, S. 91) eine wichtige Grundlage: „Ohne veränderte Narrative, Leitbilder oder Metaerzählungen, die die Zukunft von Wirtschaft und Gesellschaft neu beschreiben, kann es keine gestaltete Große Transformation geben.“

Bei einigen Autor*innen wird der Begriff Transformation von Transition abgegrenzt, wobei ersterer die umfassende bereichsübergreifende Veränderung beschreibt (Kemp und van Lente 2013) während letzterer bereits die Veränderungen von lediglich einzelnen sozialen Bereichen wie Mobilität oder den Energiesektor meint (Frantzeskaki et al. 2012, S. 20; Kemp und van Lente 2013). Bei anderen wiederum wird der Begriff Transformation für Veränderungen einzelner Bereiche und die Einführung neuer Technologien verwendet (u. a. Kemp und 
van Lente 2013; Nevens et al. 2013). Im folgenden Abschnitt werden einige Forschungsperspektiven sowie die jeweils vorhandenen Transformationsverständnisse vorgestellt.

\subsection{Forschungsperspektiven über und für Transformation}

\subsubsection{Transdisziplinäre Ansätze und Transition-Forschung}

Vor dem Hintergrund der genannten globalen Herausforderungen fordern einige Wissenschaftler*innen auch eine Neuorientierung der Forschung. Gibbons et al. (1994) und Nowotny et al. (2001) sehen in einer immer komplexer werdenden Welt, in der Wissenschaft nicht mehr in einem unabhängigem Feld agiert und Wissensproduktion in immer mehr Bereichen der Gesellschaft stattfindet, die Notwendigkeit einer neuen Form von Wissenschaft. Das Ziel dieser sogenannten Modus-2-Wissenschaft ist es, sozial robustes Wissen zu schaffen, was unter anderem durch die Einbettung in den Kontext, in dem das Wissen erzeugt wird, und die Einbeziehung von Wissen aus der sozialen Praxis erreicht werden kann. Damit grenzt sie sich von der klassischen Modus-1-Wissenschaft ab. Eine Modus-2-Universität wäre dann daraus schlussfolgernd

,$[\ldots]$ more of a synergistic institution - in a double sense. First, it may be necessary to delineate, and so demarcate, its activities according to anachronistic divisions between research and teaching, scientific and social roles. [...] Second, and much more difficult, the universities will have to acquiesce in a process of de-institutionalization, because in a Mode- 2 society the boundaries 'inside' and 'outside' make no better sense than those between research and teaching." (Nowotny et al. 2001, S. 91)

Eine Forschungsperspektive, die sich der Mode-2-Wissenschaft zuordnen lässt, ist die transdisziplinäre Forschung. Diese setzt auf die Wissensintegration zwischen Wissenschaftler*innen und Praxisakteur*innen mit dem Ziel, Lösungen für realweltliche Probleme zu entwickeln (Scholz 2017; R. Seidl et al. 2013). Wissenschaft wird dabei als öffentliches Gut angesehen, das der Gesellschaft nützlich sein sollte: Um diesem Anspruch gerecht zu werden, entwickeln Wissenschaftler*innen unterschiedlicher Disziplinen gemeinsam mit Stakeholdern Forschungsfragen und produzieren einerseits neue Erkenntnisse für die Forschung und andererseits praktische Lösungsstrategien für die Praxis (Scholz 2017, S. 7).

Eine andere Forschungsperspektive, die als Reaktion auf die sozialökologischen Herausforderungen entstand, ist die Transition-Forschung. Von zahlreichen Autor*innen dieser Forschungsperspektive wird betont, dass es sich 
bei der notwendigen Transformation - oftmals als Transition bezeichnet - um einen Prozess handelt, der eine lange Zeit, womöglich mehrere Generationen, in Anspruch nehme (Frantzeskaki et al. 2012, S. 20; Geels 2011, S. 24; Kemp und van Lente 2013, S. 116; Loorbach et al. 2017, S. 600). Andere Autor*innen (u. a. Park et al. 2012) verstehen den eigentlichen Umbruch als kurzen, weniger als zehn Jahre andauernden Prozess, der zwischen langen Phasen der Stabilität stattfindet. Auch sei der Transformationsprozess nicht überall gleich, Dauer, Art und Stärke könnten variieren (Martens und Rotmans 2005, S. 1136).

Hier zeigen sich unterschiedliche Perspektiven aus dem Bereich der TransitionForschung. Erstere beschreibt Transition oder Transformation als fundamentalen, systemübergreifenden Wandel (u. a. Kemp und van Lente 2013; Loorbach und Lijnis Huffenreuter 2013), andere als graduelle Veränderung einzelner Bereiche (u. a. Nevens et al. 2013). Einige beschreiben beide Arten von Veränderung und nutzen dafür die beiden Begriffe Transformation und Transition als unterschiedliche Prozesse (u. a. Frantzeskaki et al. 2012; Kemp und van Lente 2013).

Zahlreiche Autor*innen der Transition-Forschung (u. a. Binz et al. 2017; Fuenfschilling und Truffer 2014; Loorbach 2017; Späth und Ornetzeder 2017; Späth und Rohracher 2010) beziehen sich auf den Ansatz der Multi-LevelPerspektive (MLP) von Geels (2002, 2011). Die MLP erklärt Transformationen in einem Zusammenspiel aus Nischeninnovationen, den vorherrschenden Regimen - wie beispielsweise politischen Institutionen und Märkten - sowie externen Entwicklungen und Veränderungen auf der Ebene der Landscape. Die drei Ebenen sind durch unterschiedliche Grade der Strukturierung und der Beständigkeit gekennzeichnet, wobei sich auf Giddens (1984) Theorie der Strukturierung bezogen wird (Augenstein und Palzkill 2015, S. 5). Mit Regimen werden institutionalisierte Praktiken, Routinen sowie geteilte Werte bezeichnet (Fuenfschilling und Truffer 2014, S. 773; Geels 2011, S. 27), in denen Pfadabhängigkeiten Veränderungen erschweren (Kemp und van Lente 2013, S. 135; Loorbach et al. 2017, S. 605). Nischen sind geschützte Netzwerke von Akteur*innen, in denen Ideen und Innovationen entstehen können. Dazu zählen beispielsweise geförderte Pilotprojekte, Start-ups oder Entwicklungsabteilungen von Unternehmen (Geels 2011) oder auch Graswurzelbewegungen, die alternative Lebensstile und Konsummuster erproben (Seyfang und Smith 2007). Im Sinne von Experimenten werden diese Innovationen entwickelt, vorangebracht und verbreitet. Von Wirth et al. (2019) unterscheiden dabei drei idealtypische Formen der Diffusion: dem Einbetten der Experimente in bestehende Strukturen, der Übersetzung und Replizierung in anderen Kontexte und der Skalierung als Vergrößerung der geografischen und thematischen Verbreitung, der involvierten Akteur*innen und vorhandenen Ressourcen. Unklar bleibt bisher jedoch weitestgehend, wie die Formen der Diffusion 
zusammenhängen, ob sie alle für eine Transformation notwendig sind und unter welchen Rahmenbedingungen sie erfolgreich sind.

In den meisten Studien werden zivilgesellschaftliche Gruppen oder einzelne Unternehmen betrachtet, aber Nischenakteur*innen können auch aus anderen Bereichen, wie Politik oder Verwaltung kommen (Avelino und Wittmayer 2016, S. 638 f.). Die Stärke der Nischen wird darin gesehen, dass es dort Raum für Experimente und neue Praktiken gibt (u. a. Loorbach 2017, S. 195 f.; Seyfang und Smith 2007, S. 588).

Bei einigen dieser Innovationen gelingt es, ein Gelegenheitsfenster zu nutzen und aus der Nische heraus in die Regime zu diffundieren und diese zu verändern. Diese Gelegenheitsfenster können sich auftun, wenn durch äußere Entwicklungen in der Landscape Druck auf die bestehenden Regime ausgeübt wird (Geels 2011, S. 27 f.; Seyfang und Smith 2007, S. 589). Die entscheidende Rolle von Nischen wird also insbesondere in der Zeit des Umbruchs gesehen, wenn Regime bereits durch äußere Ereignisse und Entwicklungen destabilisiert sind (Loorbach 2017, S. 193-195). Dabei ist die Chance einer Nischeninnovation auf erfolgreiche Diffusion insbesondere dann hoch, wenn sie ein Problem anspricht, das zu der Zeit von vielen wahrgenommen wird (Seyfang und Smith 2007, S. 589). So spielt auch die öffentliche Diskussion eine Rolle dafür, in welche Richtung sich ein Regime verändert (Loorbach et al. 2017, S. 614).

Um Innovationen erfolgreich von der Nische in das Regime zu überführen und Gelegenheitsfenster nutzen zu können, müssen die Nischenakteur*innen laut Kemp und van Lente (2013, S. 135) über intensive und breite Netzwerke verfügen, die mit Ressourcen ausgestattet sind, dieselben Erwartungen in Bezug auf die Innovationen teilen und einen Prozess gemeinsamen Lernens erfahren. Brown et al. (2013, S. 703) argumentieren außerdem, dass für eine erfolgreiche Transformation sowohl formelle als auch informelle Netzwerke hilfreich sind, da beide unterschiedliche Funktionen erfüllen, die Nischeninnovationen voranzubringen. Daneben ist ein gewisser Abstand zu den Regimen und Freiraum zum Experimentieren förderlich (Fuenfschilling und Truffer 2014, S. 773). Einige Autor*innen beschäftigen sich auch mit Prozessen der Vereinnahmung der Nischeninnovationen durch die Regime. Hierbei nutzen die Regime ausgewählte Innovationen zum Selbsterhalt und um größere Veränderungen zu verhindern. Während einige Autor*innen in der Vereinnahmung vor allem Nachteile für die Nischeninnovationen sehen (u. a. Sievers-Glotzbach und Tschersich 2019, S. 7-9), betonen andere auch Vorteile für die Nischen und beobachten sich abwechselnde Prozesse der Vereinnahmung und Distanzierung (u. a. Bauler et al. 2017, S. 91; Pel 2016).

Auch wenn die MLP den Ursprung und wichtigsten Faktor einer Transformation nicht allein bei den Nischen sieht und es nicht nur um technologische 
Innovationen geht, so lag der Fokus vieler Studien aus dem Bereich der Transition-Forschung bisher trotzdem hauptsächlich auf Nischen und dort verorteten Akteur*innen mit innovativen Ideen oder Technologien (Loorbach et al. 2017, S. 605, siehe u. a. in Loorbach 2017; Nevens et al. 2013; Seyfang und Smith 2007). Die Entwicklung und Diffusion neuer Paradigmen und grundlegender Strukturen spielte in den Studien bisher eine geringere Rolle als das konkrete Erproben neuer Ideen und Technologien. In diesem Zusammenhang wurden Ansätze entwickelt, wie Transformationsprozesse aus den Nischen heraus von der Praxis gestaltet und teilweise auch von der Wissenschaft mitbegleitet werden können, so unter anderem die Urban Transition Labs (Nevens et al. 2013) oder das Transition-Management (Rotmans et al. 2001). Mittlerweile wendet sich jedoch auch die Transition-Forschung vermehrt der Ebene der Regime und Veränderungen von Institutionen zu (Loorbach et al. 2017, S. 605-609). Was in der Transition-Forschung bislang jedoch unklar bleibt, ist, wodurch Innovationen gekennzeichnet sind, die Transformationspotenzial haben und wie weit diese diffundieren müssen, bis sie als mögliche Alternativen zu Regimen gelten können. Auch der Rolle der proaktiven Unterstützung durch Politik wird sich bislang kaum gewidmet.

\subsubsection{Transformative Forschung}

Etwas neuer ist der Ansatz der transformativen Forschung, der insbesondere vom WBGU (2011, S. 23) in dem Gutachten „Welt im Wandel. Gesellschaftsvertrag für eine Große Transformation" sowie von Schneidewind und Singer-Brodowski (2013) geprägt wurde und sich in das größere Feld der eben beschriebenen Forschungsansätze einfügt ${ }^{2}$. Zusammen mit der transformativen Bildung stellt die transformative Forschung eine Weiterentwicklung des Wissenschaftssystems zur transformativen Wissenschaft dar. Diese soll es ermöglichen, die Gesellschaft hin zu einer Transformation zu unterstützen.

Bei ihrer Beschreibung der transformativen Wissenschaft knüpfen Schneidewind und Singer-Brodowski (2013, S. 78-81) an Gibbons et al. (1994) und Nowotny et al. (2001) an und setzen auf deren Konzept einer Modus-2Wissenschaft (siehe Abschn. 2.2.1) eine Modus-3-Wissenschaft auf, zu der sie die

\footnotetext{
${ }^{2}$ Die Debatte über transformative Forschung spielt sich bisher hauptsächlich im deutschsprachigen Wissenschaftsdiskurs ab. In anderen nationalen Forschungskontexten gibt es zwar ähnliche Debatten, die aber größtenteils unabhängig davon ablaufen und teilweise andere Definitionen von transformativer Forschung beinhalten (u. a. Anderson und McLachlan 2016; National Science Foundation 2007; Sen 2014; Trevors et al. 2012).
} 
transformative Forschung zählen und den Fokus der Transformationsforschung so um eine zusätzliche Perspektive erweitern. In der Modus-3-Wissenschaft wird ,,[d]as Wissenschaftssystem [...] damit zum zentralen Ort reflexiver Veränderungsfähigkeit und zu einem Katalysator für gesellschaftliche Transformationsprozesse in anderen Bereichen“ (Schneidewind und Singer-Brodowski 2013, S. 80). Ihrer Einschätzung nach verharrt das Wissenschaftssystem bislang größtenteils im Modus-1; Modus-2-Wissenschaft ist in Ansätzen vorhanden, während Modus-3-Wissenschaft bisher kaum existiert.

Um die Ziele einer Modus-3-Wissenschaft zu erläutern, beziehen sich Schneidewind und Singer-Brodowski außerdem auf Konzepte verschiedener Grade von Lernprozessen von Argyris und Schön (1978) und Sterling (2010). Während Lernen ersten Grades lediglich das Optimieren von Aktivitäten und Beheben von Fehlern ohne ein Hinterfragen der dahinterstehenden Werte ist, so werden beim Lernen zweiten Grades die Handlungen und dahinterstehenden Werte reflektiert und neue Strategien angewendet. Bei dem Lernen dritten Grades, dem transformativen Lernen, werden das Handeln selbst und die dahinterstehenden Strukturen hinterfragt:

„The case for transformative learning is that learning within paradigm does not change the paradigm, whereas learning that facilitates a fundamental recognition of paradigm and enables paradigmatic reconstruction is by definition transformative" (Sterling 2010, S. 23).

In der Modus-1-Wissenschaft findet lediglich Lernen ersten Grades, also eine Optimierung von Handlungen statt. In der Modus-2-Wissenschaft werden dagegen die Ziele und Werte von Forschung und Lehre hinterfragt. In der Modus-3Wissenschaft würden darüber hinaus auch die Wissenschaftsinstitutionen selbst reflektiert und an neue Herausforderungen angepasst (Schneidewind, SingerBrodowski, und Augenstein 2016, S. 130; Schneidewind und Singer-Brodowski 2013, S. 80, 121 f.). Die transformative Wissenschaft soll also als Modus-3Wissenschaft bewusst Transformationsprozesse hin zur Nachhaltigkeit unterstützten und initiieren, wozu teilweise neue wissenschaftliche Institutionen sowie eine Reflexion und Veränderung der bisherigen Ziele und Aktivitäten von Forschung notwendig sind, Wissenschaft sich also selbst in einen Prozess transformativen Lernens begibt.

Transformative Forschung ist eng mit der im vorangegangenen Abschnitt beschriebenen Transition-Forschung verbunden, baut dabei größtenteils auf den in diesem Kontext entwickelten Konzepten, wie der MLP (Geels 2002, 2011) 
auf und stützt sich zu einem großen Teil auf dasselbe Verständnis von Transformationsprozessen. So bezieht sich der WBGU (2011), der die transformative Forschung als Konzept vorstellt, auf die MLP und schreibt, dass „Pioniere des Wandels" in Nischen experimentieren und Innovationen vorantreiben (WBGU 2011, S. 100). Dies können kleine zivilgesellschaftliche Gruppen sein, aber auch einzelne Personen aus Ministerien, internationalen Organisationen oder Unternehmen (WBGU 2011, S. 100). Ebenfalls bezieht sich der WBGU (2011, S. 220) auf die einschränkenden Regime und hebt hervor, diese müssten so gestaltet werden, dass sie Raum für Experimente eröffnen und vielversprechende Innovationen fördern.

In Bezug auf Gelegenheitsfenster und Druck der Landscape weisen sie jedoch darauf hin, dass äußere Ereignisse zwar die Veränderungsbereitschaft in den Regimen erhöhen können, aber auch das Gegenteil bewirken und Wandel weiter erschwert werden kann (WBGU 2011, S. 101). Als förderlich für eine erfolgreiche Transformation werden das Vorhandensein von geeigneten neuen Technologien, der nötigen Finanzierung sowie eines Begleitnutzens der Neuerungen gesehen. Globale Netzwerke zum Wissensaustausch können ebenfalls hilfreich sein (WBGU 2011, S. 6). Die Verbreitung der entsprechenden Werthaltungen gegenüber der Veränderung und die Einbeziehung der Bevölkerung wird außerdem vorausgesetzt (WBGU 2011, S. 8). Daher sieht der WBGU (2011, S. 257-278) die Rolle von Nischen darin, Ideen zu entwickeln, Alternativen aufzuzeigen und einen Wertewandel zu ermöglichen, was insbesondere zu Beginn einer Transformation von großer Bedeutung ist. Um Veränderungen zu ermöglichen, müssen zur Unterstützung der Innovationen die politischen Rahmenbedingungen entsprechend angepasst und die Neuerungen institutionell verankert werden (WBGU 2011, S. 269). Hinderlich sind ökonomische, politische und institutionelle Pfadabhängigkeiten, das Fehlen von Vorbildern - beispielsweise $\mathrm{CO}_{2}$-neutraler Städte - das Vorhandensein weiterer fossiler Ressourcen sowie das enge Zeitfenster, das noch zur Verhinderung irreversibler Umweltveränderungen bleibt (WBGU 2011, S. 6). Laut WBGU hat die „Große Transformation“ bereits in den 1970er Jahren begonnen. Die zentralen Richtungsentscheidungen wurden im Gutachten des WBGU von 2011 für in den zehn folgenden Jahren notwendig und möglich beschrieben, gefolgt von einer Phase der Umsetzung der Entscheidungen und einer Stabilisierung. Insgesamt erstreckt sich die Transformation laut WBGU ( 2011, S. 1, 7) folglich über mehrere Generationen, wobei die eigentliche Phase des Umbruchs mit zehn Jahren relativ kurz andauert. Da es sich bei der "Großen Transformation“ um einen weltweiten Prozess handelt, finden parallel an verschiedenen Orten Transformationsprozesse in unterschiedlicher Form und Geschwindigkeit statt (WBGU 2011, S. 89 f.). 
Wie genau Transformationsblockaden überwunden werden können, ist laut WBGU (2011, S. 346) noch nicht genügend erforscht. Unklar bleibt aus den Ausführungen des WBGU außerdem, ob die kleinen Maßnahmen und Experimente als wichtige kleine Schritte der Transformation zu sehen sind oder ob sie vor allem den Wertewandel voranbringen, welcher notwendige Voraussetzungen für diese darstellt. Bei der Forderung des WBGU (2011, S. 220 f.) nach Unterstützung von Innovationen und alternativen Ideen durch die Politik, stellt sich die Frage, ob es realistisch ist, dass Entscheidungsträger*innen auch grundsätzliche Alternativen zum Status-quo des aktuellen Wirtschafts- und Gesellschaftssystems unterstützen.

Als Katalysatoren dieses eben beschriebenen gesellschaftlichen Wandels machen die transformativen Wissenschaftler*innen ihre Werturteile und Normen explizit (Schneidewind 2018, S. 432; Schneidewind, Singer-Brodowski, und Augenstein 2016, S. 124-129; Schneidewind und Singer-Brodowski 2013, S. 72 f.) und gehen über ihre neutrale Rolle als beobachtende Instanz hinaus, indem sie selbst als Pionier*innen des Wandels agieren (Rose et al. 2017). Dies resultiert neben der klassischen Forschung auch in zusätzlichen Tätigkeiten, so beispielsweise der Begleitung von Prozessen, der Vernetzung von Akteur*innen oder dem Initiieren von Interventionen in Reallaboren (Hilger et al. 2018). Die Forschenden entwickeln selbst oder gemeinsam mit anderen Akteur*innen innovative Lösungen und bringen diese voran (Schneidewind, Singer-Brodowski, und Augenstein 2016, S. 127-171). So schreiben Augenstein et al. (2016, S. 171) über die Rolle von Wissenschaft in diesen Prozessen:

„In urbanen Transformationssituationen können wissenschaftliche Narrative auf unterschiedlichen Ebenen Wirkungen entfalten - insbesondere, wenn sie in enger Kooperation mit den konkret in der Stadt handelnden AkteurInnen entstehen. Aus diesem Grund ist die partizipative Form von Wissenschaft ein konstituierendes Merkmal transformativer Wissenschaft.“

Transformative Forschung arbeitet also oft gemeinsam mit Nischenakteur*innen, die bereits versuchen, gesellschaftliche Veränderungen voranzubringen und so von der Wissenschaft unterstützt werden. Um diese intendierte Wirkung zu erreichen und herauszufinden, wie Transformation aktiv unterstützt werden kann, wird bei transformativer Forschung Wissen verschiedener Arten geschaffen: Systemwissen, Zielwissen und Transformationswissen (Schneidewind 2018, S. 431-433; Schneidewind und Singer-Brodowski 2013, S. 69-72; siehe auch Pohl und Hirsch Hadorn 2008). Erste Projekte, die sich im Sinne der transformativen Forschung 
verstehen, agieren vor allem auf kleinräumiger Ebene von Städten oder gar Quartieren (Schneidewind et al. 2018) und erscheinen weniger bewusst gestaltet als im Gutachten des WBGU beschrieben. Um sich mit geeigneten Nischenakteur*innen zu vernetzen, müssen sie gewissermaßen ad hoc auf Prozesse reagieren. Wie dies in den beiden hier untersuchten Fallbeispielen gelang, wird im Analyseteil des Buches (siehe Kap. 5) genauer betrachtet.

Im deutschsprachigen Wissenschaftsdiskurs hat sich im Zusammenhang mit dem Forschungsansatz der transformativen Forschung eine Debatte darüber entzündet, ob Wissenschaft sich in diese Richtung entwickeln und aktiv Transformation mitgestalten sollte. Diese Diskussion wurde vor allem in der Zeitschrift „GAIA - Ökologische Perspektiven für Wissenschaft und Gesellschaft" ausgetragen (u. a. Böschen 2014; Grunwald 2015; Kläy und Schneider 2015; Krainer und Winiwarter 2016; Lucas et al. 2013; Rohe 2015; Schneidewind 2013, 2015; von Wissel 2015) sowie vom damaligen Präsidenten der Deutschen Forschungsgemeinschaft Strohschneider (2014) in seinem Artikel „Zur Politik der Transformativen Wissenschaft" aufgegriffen.

Strohschneider (2014, S. 180) kritisiert die transformative Forschung dafür, dass sie auf Problemlösung ausgerichtet sei und in erster Linie zur Lösung realweltlicher Probleme beitragen will. Ihm zufolge sollte Forschung aber zu allererst wissenschaftliche Fragestellungen analysieren und verstehen. Auch wenn in einigen Bereichen der Wissenschaft viel angewandte Forschung betrieben wird, sollte diese die Grundlagenforschung nicht ersetzen. So argumentiert er, ,[...] dass, um es so zu sagen, Epistemologie wissenschaftlich generell durch Praxeologie substituierbar sei, dies muss bezweifelt werden“" (Strohschneider 2014, S. 180). Die Fürsprecher*innen dieses transformativen Ansatzes fordern jedoch auch nicht ein Ersetzen von Grundlagenforschung durch die anwendungsorientierte transformative Forschung, sondern sehen dies als notwendige Ergänzung (Grunwald 2015, S. 19 f.). Daneben schafft die transdisziplinäre Forschung, die Strohschneider (2014) hier ebenfalls kritisiert, stets auch Erkenntnisgewinne für die Wissenschaft und nicht lediglich Lösungsansätze für die Praxis (Scholz 2017, S. 7). Auch wenn bei transformativer Forschung der Fokus auf praktischen Lösungsansätzen liegt, wird auch eine Relevanz der Ergebnisse für die Wissenschaft erwartet, so beispielsweise bei Reallaboren (Wanner et al. 2018) und sowohl Zielund Transformations- als auch Systemwissen produziert (Schneidewind 2018, S. 430 f.; Schneidewind und Singer-Brodowski 2013, S. 123). Trotzdem ist dieses stark kontextualisierte Wissen oftmals kaum generalisierbar (Schneidewind und Singer-Brodowski 2013, S. 123).

An dem transdisziplinären Ansatz transformativer Forschung kritisiert Strohschneider (2014, S. 180 f.) außerdem, dass außerhalb der Wissenschaft darüber 
entschieden werde, was als wissenschaftliche Fragestellung verstanden und was wissenschaftlich bearbeitet werden soll. Auch die Kategorisierung von Ziel-, System- und Transformationswissen sieht er kritisch. Dadurch würde die Grenze zwischen Gesellschaft und Wissenschaft verschwimmen. Der ohnehin stattfindenden „Verwissenschaftlichung moderner Gesellschaften“ würde die transformative Wissenschaft die „Vergesellschaftung von Wissenschaft“ entgegensetzen, wobei er beide Entwicklungen kritisch sieht (Strohschneider 2014, S. 182). Scholz (2017, S. 12) entgegnet diesen Ausführungen, dass sowohl transdisziplinäre als auch transformative Forschung die Qualitätskriterien der Wissenschaft, die Eigenständigkeit von Forschung und die Unterscheidbarkeit von Gesellschaft und Wissenschaft nicht in Frage stellen. Außerdem werden Forschungsfragen nicht lediglich von der Praxis diktiert, sondern gemeinsam zwischen Wissenschaft und Praxis erarbeitet (Asayama et al. 2019; Mauser et al. 2013).

Auch wirft Strohschneider (2014, S. 181) der transformativen Forschung vor, die klassischerweise in der Wissenschaft geltende Dichotomie zwischen wahr und unwahr durch nützlich und nicht nützlich für Nachhaltigkeit ersetzen zu wollen. Nachhaltigkeit würde durch die transformative Wissenschaft als letztendliches normatives Ziel andere Ziele ausblenden. Dagegen läge es nicht in der Kompetenz der Wissenschaft, Entscheidungen über eine Transformation zu treffen, da dies politische Entscheidungen seien, die die Bevölkerung betreffen. Scholz (2017, S. 12) argumentiert jedoch, dass die Transformation hin zu einer ressourcenschonenden Gesellschaft schon als demokratisch legitimiert angesehen werden kann, da sie in mehreren internationalen Verträgen formuliert wurde. Bei der grundsätzlichen Zielrichtung handelt es sich also nicht um eine ausschließlich von den Forschenden gesetzte subjektive Einschätzung.

Bei einigen Kritikpunkten Strohschneiders handelt es sich also um Missverständnisse, so bei der Annahme, dass die Modus-3-Wissenschaft die anderen Aspekte von Wissenschaft ersetzen soll. Andere Kritikpunkte deuten auf zwei grundsätzlich unterschiedliche Verständnisse davon hin, inwieweit sich Wissenschaft in gesellschaftliche und politische Prozesse einbringen darf oder gar sollte und wie sehr sie dabei der Objektivität verpflichtet ist. Die Vertreter*innen der transformativen Forschung sehen es gerade als ihre Aufgabe an, ihr Wissen der Gesellschaft zur Verfügung zu stellen und den Weg hin zu einer Nachhaltigkeitstransformation zu unterstützen (Schneidewind 2015, S. 88; Schneidewind und Singer-Brodowski 2013, S. 68; WBGU 2011, S. 342 f.).

Was bei der transformativen Forschung und dem dahinterliegenden Verständnis von Transformationsprozessen jedoch tatsächlich bislang unklar ist, ist wie die kleinteiligen Maßnahmen und Innovationen - ob von Forschenden oder anderen Pionier*innen des Wandels - zusammenspielen und welche Rolle kleinräumige 
Prozesse beispielsweise in Städten für die „Große Transformation“ spielen. Im folgenden Abschnitt werden zu diesem letztgenannten Punkt wissenschaftliche Erkenntnisse anderer Forschungsgebiete hinzugezogen.

\subsection{Städte als Orte der Transformation}

Inwieweit in Städten überhaupt eigene Politikentscheidungen getroffen werden und neue Ideen in städtischen Diskursen einen Einfluss auf darüberliegende Prozesse haben können, soll in den nächsten Absätzen mithilfe von Literatur aus den Bereichen Stadtsoziologie, Kommunalpolitik und urbaner Nachhaltigkeitstransformation dargestellt werden. Städte haben in einigen Bereichen einen Handlungsspielraum, in anderen Bereichen setzen sie vor allem die auf höheren Politikebenen gefällten Entscheidungen um.

Städte haben die „Alleinzuständigkeit“ bei allen örtlichen Angelegenheiten, die nur durch explizit im Grundgesetz genannte Kompetenzen von Ländern oder dem Bund eingeschränkt werden und haben ein Recht auf Selbstverwaltung. Außerdem müssen sie Bundes- und Landesgesetze umsetzen (Häußermann et al. 2008, S. 331; Wollmann 2008). Die Einschränkungen der Kompetenzen sind aber mittlerweile sehr weitreichend und zusätzlich indirekt durch die finanziellen Abhängigkeiten und Verflechtungen sowie die knappe Haushaltslage vieler Kommunen limitiert (Häußermann et al. 2008, S. 331). Häußermann et al. (2008, S. 331) beschreiben die Situation zwiegespalten:

„Die Kommunen haben daher eine eigentümliche Stellung im politisch-administrativen System: Sie sind Körperschaften des öffentlichen Rechts, also nicht Teil des Staates, aber sie können auch nicht selbstständig handeln, weil sie einerseits staatliche Aufgaben zu erledigen [haben] und andererseits finanziell davon abhängig sind, welche Einkommensquellen ihnen von Bund und Ländern zugewiesen werden.“

Doch auch wenn der Spielraum für Entscheidungen limitiert ist, so werden die Tätigkeiten von Kommunen laut Vetter und Holtkamp (2008, S. 19) von vielen Bürger*innen teilweise als relevanter für sie persönlich wahrgenommen als nationale oder europäische Politik. So ist die lokale Politik näher an ihrer Alltagswelt und kann in Ansätzen kompensieren, dass durch Europäisierung und Globalisierung viele Bürger*innen die Politik insgesamt entfernt von ihrer Lebensrealität wahrnehmen (Vetter und Holtkamp 2008, S. 19). Dadurch können Kommunen durch ihre Nähe zu örtlichen Gegebenheiten teilweise besser, effektiver und effizienter öffentliche Mittel verteilen, da sie wissen, was an welcher Stelle benötigt 
wird (Vetter und Holtkamp 2008, S. 19). In diesem Zusammenhang hat auch Bürgerbeteiligung in Städten in der Vergangenheit an Bedeutung gewonnen und alle Bundesländer haben Regelungen für Bürgerentscheide oder Bürgerbegehren auf der Ebene von Städten (Häußermann et al. 2008, S. 334).

Die Aufgaben von Kommunen lassen sich in vier Bereiche differenzieren (Häußermann et al. 2008, S. 335): Über die freiwilligen Tätigkeiten - wie kulturelle Aktivitäten, Sport- und Freizeiteinrichtungen und Integrationsleistungen - können die Kommunen selbstständig entscheiden. Bei den Pflichtaufgaben ohne Weisung sind die Aufgaben festgelegt, nicht aber die Details der Umsetzung. Pflichtaufgaben nach Weisung sind dagegen auch bezüglich der Umsetzung genau festgelegt. Daneben gibt es staatliche Auftragsangelegenheiten. Bei einigen Themen können die städtischen Entscheidungsträger*innen also kaum Einfluss nehmen, in anderen Bereichen haben sie jedoch durchaus Handlungsspielraum. So sehen Häußermann et al. (2008, S. 341) den Einfluss von Städten insbesondere in den Bereichen Wohnungsbau, städtische Infrastruktur, insbesondere die Verkehrsinfrastruktur, Kultur und Handel. Je besser die Haushaltslage einer Stadt ist, umso mehr können zusätzlich zu den Pflichtaufgaben noch freiwillige Aufgaben übernommen werden. Bei knapper Finanzlage bleibt hier wenig Handlungsspielraum (Häußermann et al. 2008, S. 335; Kost 2010). So beobachtet auch Kost (2010, S. 234):

„Für Städte und Gemeinden wird es immer schwieriger, politische Gestaltungsräume zu eröffnen, weil durch die wirtschaftliche Krisensituation die dramatisch zunehmenden finanziellen Belastungen die Kommunen immer stärker auf die Erfüllung ihrer von höherer Ebene zugewiesenen Pflichtaufgaben beschränken und an den Rand ihrer Handlungsfähigkeit führen.“

In vielen Bereichen kann lokale Politik dennoch mehr gestalten und nicht nur Gesetze der Landes-, Bundes- und EU-Ebene umsetzen. Insbesondere wird dies daran deutlich, dass ähnliche Probleme in verschiedenen Städten unterschiedlich angegangen werden (Zimmermann 2008, S. 211-213). Dies gilt nicht nur für Kommunen in Deutschland, sondern auch darüber hinaus, was unter anderem auch von Autor*innen der Transition-Forschung betrachtet wird. So zeigt das Beispiel Kopenhagen mit seinen zahlreichen umweltpolitischen Maßnahmen und seinem Ziel der $\mathrm{CO}_{2}$-Neutralität bis 2025, dass Städte beim Thema Nachhaltigkeitstransformation durchaus eigenständig agieren und unterschiedliche Lösungsansätze entwickeln und umsetzen können (Frantzeskaki et al. 2017, S. 1). Daneben werden viele neue Innovationen in Städten entwickelt und getestet (Frantzeskaki et al. 2017, S. 1 f.; Loorbach und Shiroyama 2016, S. 4). In Städten, 
so Fuenfschilling und Truffer (2014, S. 773), verschwimmen teilweise die Grenzen zwischen Nischenakteur*innen mit innovativen Ideen auf der einen und den Regimen auf der anderen Seite. Durch räumliche Nähe kommt es insbesondere in Städten zu Netzwerken und personellen Überschneidungen zwischen den beiden Bereichen.

Städte werden als einschränkende aber auch förderliche Strukturen für Wandel beschrieben. Auf der einen Seite erschweren stark institutionalisierte Strukturen von Städten und damit zusammenhängende Pfadabhängigkeit Wandel. Auf der anderen Seite können bestimmte Strukturen aber auch förderlich für Wandel sein und Städte als strategische Nischen dienen, in denen Best-Practice-Beispiele entwickelt werden (Fuenfschilling 2017, S. 153). Dies geschieht insbesondere auch im Bereich Klimaschutz, wo Städte in den Fokus rücken, weil nationale Regierungen bisher keine ausreichenden Schritte ergriffen haben (Rohracher und Späth 2017, S. 288). Da in Städten ökologische, sozio-ökonomische sowie politische Krisen jedoch besonders spürbar werden, ist hier in einigen Fällen der Handlungsdruck höher (Loorbach und Shiroyama 2016, S. 4).

Dieser unterschiedliche Umgang von Städten mit Krisen und mit aufkommenden Innovationen kann auf spezifische lokale Gegebenheiten zurückgeführt werden. Löw (2008, S. 77) beschreibt dies als „Eigenlogik“, als „die verborgenen Strukturen der Städte als vor Ort eingespielte, zumeist stillschweigend wirksame präreflexive Prozesse der Sinnkonstruktion (Doxa) und ihrer körperlich-kognitiven Einschreibung (Habitus)“. Sie und andere Autor*innen weisen damit darauf hin, dass Städte durchaus abgrenzbare Entitäten sind, in denen sich Prozesse unterschiedlich entwickeln und die auf äußere Einflüsse unterschiedlich reagieren (Löw 2008, S. 73-83, 2012; Zimmermann 2008, S. 208). Auch der WBGU (2016, S. 153 f.) beschreibt mit dem Konzept „Eigenart“ einen ähnlichen Aspekt. Die Eigenlogik einer Stadt ist unter anderem bestimmt durch die jeweilige historische, kulturelle und ökonomische Prägung:

„Vielmehr existiert eine routinisierte und habitualisierte Praxis (verstanden als strukturierte und strukturierende Handlungen), die ortsspezifisch im Rückgriff auf historische Ereignisse, materielle Substanz, technologische Produkte, kulturelle Praktiken sowie ökonomische oder politische Figurationen (und deren Zusammenspiel) abläuft." (Löw 2008, S. 77)

Barbehön et al. (2015) und Barbehön und Münch (2017) untersuchen städtische Diskurse und zeigen, dass ähnliche Probleme unterschiedliche Krisendefinitionen hervorrufen, die sie der Eigenlogik der Städte zuschreiben. Diese Analysen 
von städtischen Krisendefinitionen zeigen, dass in Städten durchaus eigene Diskursarenen existieren, die nicht völlig losgelöst, doch trotzdem unterschiedlich ablaufen. So können sowohl spezifische Krisendefinitionen entstehen als auch unterschiedliche Lösungsansätze formuliert und umgesetzt werden. Zimmermann (2008, S. 214) beschreibt Eigenlogik als kontextspezifisches Politikmuster und meint, die Ausprägung der Eigenlogik hänge

,[...] entscheidend von den örtlich differierenden Wahrnehmungen und Interpretationen der gegenwärtigen sozialen und ökonomischen Umstrukturierungen, den unterschiedlichen Konflikt- und Konsensbildungsprozessen in den lokalen Akteursnetzwerken sowie von den jeweiligen sozio-kulturellen Kontexten und Milieus ab [...].“

$\mathrm{Zu}$ bestimmten Themen kann also auch in Städten ein abgrenzbarer Diskurs entstehen, der natürlich von Diskursen und Vorkommnissen von außerhalb der Stadt beeinflusst wird. Dies trifft jedoch auch auf nationale Diskurse zu, die insbesondere in Zeiten fortgeschrittener Globalisierung und Europäisierung ebenfalls nicht komplett losgelöst von Diskursen anderer Länder oder darüber liegender Politikebenen sind (Alasuutari 2015, S. 165; Löw 2008, S. 122-132). Trotz unterschiedlicher Problemwahrnehmungen und Lösungsansätze beobachtet Häußermann (2008, S. 341 f.) bei allen Kommunen, dass ,,[...] sich eine Stadtverwaltung um wirtschaftliches Wachstum bemühen und damit das langfristige Interesse der Stadt verfolgen muß, darüber gibt es nirgends Kontroversen" und spricht hiermit indirekt das vorherrschende Paradigma von Wirtschaftswachstum an.

Um die vorherrschenden Paradigmen, Krisenwahrnehmungen oder andere Inhalte vorhandener Diskurse aufzudecken, betrachten viele Autor*innen (u. a. Barbehön et al. 2015; Gardner 2017; Romsdahl et al. 2017) in ihren Analysen insbesondere lokale Presseartikel, Protokolle von Ratssitzungen, Webseiten der Städte und Interviews mit Entscheidungsträger*innen und definieren diese Dokumente somit als relevante Teile städtischer Diskurse.

Aus der in den vorangegangenen Absätzen genannten Literatur lässt sich insgesamt schließen, dass politische Entscheidungen zu den gleichen Fragestellungen in verschiedenen Städten durchaus unterschiedlich sein können und von den Rahmenbedingungen in der jeweiligen Stadt abhängig sind. Durch die spezifische Eigenart, die Konstellation der Akteur*innen sowie die vorherrschenden Diskurse können Städte so in der Analyse als abgrenzbare Einheiten betrachtet werden. Es wird davon ausgegangen, dass auf der Ebene einer Stadt lokale 
Akteur*innen wie beispielsweise Entscheidungsträger*innen sowie zivilgesellschaftliche Akteur*innen und Wissenschaftler*innen spezifische Diskurse einer Stadt sowie politische Entscheidungen mitgestalten, indem sie Ideen voranbringen. Dies kann durch Präsenz in lokalen Medien, sozialen Netzwerken oder Ratssitzungen, Demonstrationen und Bürgerinitiativen geschehen. Durch eine bestimmte Konstellation von Ideen und Akteur*innen, so wird angenommen, kann es dabei auch zu Politikveränderungen kommen.

\subsection{Institutioneller Kontext in Wuppertal}

Da wie eben erwähnt aufgrund der Eigenlogik (Löw 2008, 2012) beziehungsweise Eigenart (WBGU 2016) von Städten Veränderungen in verschiedenen Städten unterschiedlich verlaufen und von den örtlichen Begebenheiten und Akteur*innen abhängen, wird im Folgenden ein Überblick über den Kontext in der Stadt Wuppertal während des Untersuchungszeitraumes sowie wichtige Entwicklungen davor gegeben. Fokus liegt dabei auf institutionellen Strukturen, aktuellen Herausforderungen und vorherrschenden Narrativen.

Wuppertal wurde im 18. und 19. Jahrhundert zu einem wichtigen Industriezentrum, insbesondere der Textilproduktion, jedoch auch von Werkzeugen und anderen Waren. In diesem Kontext entstanden zahlreiche Innovationen und es entwickelte sich zunehmender Wohlstand unter einem Teil der Bevölkerung. Das Angebot an Arbeitsplätzen sorgte für einen Zuzug zahlreicher Menschen, so dass die Bevölkerungszahlen stark wuchsen. Die Orte, die heute Teil der Stadt Wuppertal sind, gehörten am Ende des 19. Jahrhunderts zu den dichtest besiedelten Regionen in Preußen und größten Stadtregionen Deutschlands (Wittmütz 2013, S. 37-75).

Gleichzeitig erlebte die Stadt jedoch auch frühe Erfahrungen mit sozialen Problemen, insbesondere hohe Ungleichheit, Armut durch niedrige Löhne und schlechte Wohnbedingungen der armen Arbeiterklasse. Die Bevölkerungszahlen wuchsen schneller als neue Wohnungen gebaut wurden und trotz zahlreicher Versuche konnte dieses Problem lange Zeit nicht endgültig gelöst werden (De Buhr 1984, S. 58-62; Wittmütz 2013, S. 75-100).

Seit Beginn des 19. Jahrhunderts wurden insbesondere von der oberen Mittelschicht zahlreiche Bürgervereinigungen gegründet, die gute Kontakte zur Stadtverwaltung hatten und ihren Einfluss auf lokale Entscheidungen steigern konnten (Heinrichs 1984, S. 109-112). Einige Zeit später gründeten sich auch Vereine der Arbeiterklasse, die Entwicklungen zu beeinflussen versuchten sowie sich insbesondere dem Freizeitsektor widmeten (Heinrichs 1984, S. 116). 
Ab den 1970er Jahren sank wie in anderen ehemaligen Industriezentren auch in Wuppertal im Zuge des Strukturwandels die Bevölkerungszahl wegen Abwanderung stark (IT.NRW o. J.) und Wuppertal hat bis heute mit den Folgen der Schließung zahlreicher Fabriken und hoher Arbeitslosigkeitsquote unter den Gebliebenen zu kämpfen. Bis heute ist die hohe Leerstandsquote von Wohnungen im Stadtbild prägend (2013: 6,6 \%, was 12.950 Wohnungen entspricht, Stadt Wuppertal 2015, S. 4). Aktuell steigt die Bevölkerungszahl jedoch wieder leicht an, was vor allem auf den Zuzug von Menschen aus dem Ausland zurückzuführen ist (IHK 2015).

Die Arbeitslosigkeitsquote war mit 9,6 \% im Jahr 2015 im Vergleich zu anderen deutschen Städten hoch, war jedoch in den vorangegangenen Jahren leicht gesunken (IHK 2015). Die Kommune ist seit Jahren hoch verschuldet. 2015, zu Beginn des Untersuchungszeitraumes, beliefen sich diese Schulden auf 1,9 Milliarden Euro (IT NRW 2016) - und auch sehr viele private Haushalte sind verschuldet (Reutter et al. 2012). In Städterankings schneidet Wuppertal meist schlecht ab, so steht es im HWWI/Berenberg-Städteranking 2015 auf Platz 29 von 30, im Prognos Zukunftsatlas 2016 auf Platz 231 von 402 der Kreise und kreisfreien Städte Deutschlands (Nitt-Drießelmann und Wedemeiner 2015; Prognos AG 2016).

Die darin abgebildete wirtschaftliche Entwicklung und Wettbewerbsfähigkeit als Wohnort spielen auch in den Stadtentwicklungsstrategien und Leitlinien der Stadt Wuppertal eine zentrale Rolle. So beziehen sich ein Großteil der den „Leitlinien der Wuppertaler Stadtentwicklung 2015“ zugeordneten Projekte auf die Ziele „Stärkung der Wirtschafts- und Innovationskraft Wuppertals“ und „Schaffung von Arbeits- und Ausbildungsplätzen“ (Oberbürgermeister der Stadt Wuppertal 2008). Die genannten Leitlinien sind jedoch insgesamt breiter aufgestellt und haben eine Förderung von Bildung, Kultur und Freizeit, Sozialem sowie Umweltaspekte zum Ziel.

Ähnlich hat es die Stadtentwicklungsstrategie W2025 als Fortschreibung der Leitlinien 2015 zum Ziel, die Lebensqualität in Wuppertal allgemein zu steigern und bezieht sich dabei auf die lange Phase der Kürzungen und finanziellen Engpässe der Stadt infolge hoher kommunaler Schulden. 13 Schlüsselprojekte sind hier auf unterschiedliche Bereiche verteilt, Hauptevaluierungsinstrument ist dann jedoch das Städteranking der Wirtschaftswoche. Laut W2025 ist die primäre Zielgruppe der Stadtentwicklungsstrategie die Einwohnerschaft. Als sekundäre Zielgruppe wird dann direkt die Wirtschaft genannt, da 
„die materiellen Indikatoren der Lebensqualität [...] durch eine gesunde Wirtschaftslage gesichert [werden]. Sie garantiert die Einkommen der Bürgerinnen und Bürger, die wiederum die Einnahmen der Stadt sichern“" (Stadt Wuppertal 2013, S. 3).

Der Koalitionsvertrag der Wuppertaler Ratsfraktionen der Sozialdemokratischen Partei Deutschlands (SPD) und der Christlich Demokratischen Union Deutschlands (CDU) im Nachgang zu der Kommunalwahl 2014 beschreibt in der Präambel

„,[d]ie Aufrechterhaltung der finanzpolitischen Handlungsfähigkeit der Stadt Wuppertal [als] [...] eine der Kernaufgaben unserer gemeinsamen Politik für diese Ratsperiode. Vor diesem Hintergrund stehen sämtliche Vorhaben zunächst einmal unter dem Vorbehalt der Finanzierbarkeit“" (SPD/CDU 2014, S. 2).

Auch hier fallen die Stichworte des Wirtschaftsstandortes Wuppertals und der Familienfreundlichkeit im Wettbewerb mit anderen Städten als Wohnort. Diese Fokussierung auf einen ausgeglichenen Haushalt hängt maßgeblich mit dem Stärkungspaktgesetz zusammen, durch das verschuldete Kommunen finanzielle Unterstützung bekommen, im Gegenzug jedoch selbst Anstrengungen unternehmen müssen, um den Haushalt auszugleichen (SPD/CDU 2014, S. 2-4).

Es kann also davon ausgegangen werden, dass die Stadtpolitik und -verwaltung zwar ein ganzheitliches Bild von Lebensqualität in ihren Programmen vertritt, bei konkreten Projekten und Entwicklungen dann jedoch meist ökonomische Aspekte in den Vordergrund stellt, da davon ausgegangen wird, dass diese die Grundlage für andere Bereiche der Lebensqualität sind. Dahinterliegendes Paradigma scheint es zu sein, dass Wirtschaftswachstum die Lebensqualität steigert und eine Stadt sich im Wettbewerb mit anderen behaupten muss. Dabei wird vor allem auf die Gewinnung von Investoren und das Schaffen von Arbeitsplätzen gesetzt und kaum alternative Formen regionalen oder nachhaltigen Wirtschaftens erwähnt.

Doch trotz oder gerade wegen der schwierigen finanziellen Lage in Wuppertal verfügt die Stadt über eine sehr aktive Zivilgesellschaft. Viele der im 19. Jahrhundert gegründeten Bürgervereine sind heute noch aktiv, insbesondere die Quartiersvereine, welche in fast allen Quartieren der Stadt vorhanden sind und sich für die Verbesserung des lokalen Lebens und der Nachbarschaft einsetzen. Außerdem gibt es zahlreiche stadtweite Vereine und Initiativen, die sich für einzelne Themen einsetzen, wie beispielsweise die Verbesserung des Radverkehrs oder die Parkanlagen der Stadt. Zusätzlich zu diesen thematisch ausgerichteten Initiativen und Quartiersvereinen wurden in den vergangenen Jahren zahlreiche 
Kulturzentren gegründet und urbane Gärten und soziale Projekte auf Brachflächen umgesetzt. Wuppertal zeichnet sich also durch eine hohe Anzahl und Varietät von zivilgesellschaftlichen Gruppen aus, die meist gut miteinander vernetzt sind. In vielen Bereichen konnte die aktive Zivilgesellschaft in den vergangenen Jahren bereits viel erreichen, so beispielsweise den Bau einer Fahrrad- und Fußverbindung zwischen dem östlichen und westlichen Rand der Stadt auf einer ehemals brachliegenden Zugtrasse, der Nordbahntrasse (Wuppertalbewegung e. V. o. J.).

Auch in der Stadtverwaltung und -politik gibt es Veränderungen der letzten Jahre zu verzeichnen. So hatte Wuppertal von Mitte 2016 bis 2017 als erste deutsche Stadt ein Beteiligungsdezernat. Dieses wurde zwar 2017 mit Abwahl des Dezernenten wieder aufgelöst, jedoch in eine Stabsstelle für Bürgerbeteiligung umgewandelt. Diese hat zum Ziel, den Dialog zwischen Bürgerschaft und Stadtpolitik und -verwaltung zu verbessern (Stadt Wuppertal 2016). Von Beginn an gab es von Seiten der Bürgerschaft und Politik Kritik an der Schaffung des neuen Dezernates sowie hohe Erwartungen schneller und spürbarer Veränderungen der Beziehung zwischen Stadtrat, -verwaltung und -gesellschaft. Dass an anderen Stellen durch die hohen Kosten des zusätzlichen Dezernates Geld gekürzt wurde, stieß auf viel Kritik und Unverständnis (WZ 2014, 2015a, 2015b, 2016).

Zusätzlich zur aktiven Zivilgesellschaft und den Veränderungen in der Stadtverwaltung ist Wuppertal außerdem geprägt von den Forschungsinstitutionen, die dort ihren Standort haben. Das WI beschäftigt sich seit 1991 mit verschiedenen Themen im Bereich Umwelt und Nachhaltigkeit und vermehrt auch mit den Entwicklungen in der unmittelbaren Region (WI o. J.). Die BUW wurde 1972 als Gesamthochschule gegründet und ist seitdem stetig gewachsen (BUW o. J.). Im Jahr 2013 gründeten das WI und die BUW gemeinsam das Transzent und institutionalisierten so ihre enge Zusammenarbeit in den Themenbereichen Nachhaltigkeit und sozial-ökologische Transformation. Dieses Forschungszentrum vereint die anwendungsorientierte Forschung des WI mit der universitären Forschung und Lehre (Transzent 2019).

Insgesamt kann Wuppertal also als Stadt in einer schwierigen finanziellen und sozialen Lage bezeichnet werden, die jedoch aktuell einige positive Veränderungen verzeichnet und die von einer sehr aktiven Bürgerschaft charakterisiert ist. Daneben gibt es durch die Forschungseinrichtungen weitere Akteur*innen, die eine nachhaltige Stadtentwicklung voranzutreiben versuchen und neue Ideen in die Stadt bringen. Auf die verschiedenen institutionellen Veränderungen im Laufe des Untersuchungszeitraumes wird im Rahmen der Analyse an einigen Stellen Bezug genommen. 


\subsection{Zwischenfazit: Zusammenführung des für die Analyse relevanten Hintergrundes}

In den vorangegangenen Abschnitten wurden die Hintergründe erläutert, die für die vorliegende Arbeit relevant sind: Ein kurzer Überblick über die Forderungen nachhaltiger Entwicklung und einer Transformation von Politik und Gesellschaft, um irreversible Umweltveränderungen aufzuhalten (Abschn. 2.1); die transformative Forschung sowie weitere verwandte Forschungsperspektiven, die als Reaktion darauf entstanden sind (Abschn. 2.2) sowie die Handlungsmöglichkeiten von Städten bei diesen Transformationsprozessen (Abschn. 2.3). Zuletzt wurde der institutionelle Kontext der Stadt Wuppertal, in dem beide im späteren Verlauf analysierten Fallbeispiele zu verorten sind, vorgestellt.

Hierbei hat sich gezeigt, dass Wuppertal als hochverschuldete Stadt weiterhin einen stadtpolitischen Fokus auf die ökonomische Entwicklung, Wettbewerbsfähigkeit sowie Arbeitsplätze legt. Die knappe Haushaltslage erschwert womöglich den eigenen Handlungsspielraum der Kommune, der wie erläutert (Abschn. 2.3) von finanziellen Mitteln abhängt (Häußermann et al. 2008, S. 335; Kost 2010). Doch gleichzeitig sind zahlreiche Akteur*innen aus Wissenschaft und Zivilgesellschaft und teilweise Stadtverwaltung an Veränderungen in Richtung Nachhaltigkeit interessiert und setzen sich für eine Verbesserung der Lebensqualität in der Stadt Wuppertal oder ihrer Nachbarschaft ein.

Eine Notwendigkeit dieser Veränderung in Richtung Nachhaltigkeit wurde in den vergangenen Jahren und Jahrzehnten immer deutlicher, da planetare Leitplanken bald erreicht oder gar bereits überschritten sind (Rockström et al. 2009; Steffen et al. 2015) und irreversible Umweltschäden zeitnah verhindert werden müssen (siehe Abschn. 2.1). Dazu ist eine umfassende Veränderung - eine „Große Transformation“ - notwendig (WBGU 2011), die auch die Überwindung des auf andauerndem Wachstum und dem Verbrauch fossiler Ressourcen basierenden Wirtschaftssystems und den damit zusammenhängenden Paradigmen beinhaltet.

Eine prominente Forschungsperspektive, die sich mit diesen Transformationsprozessen beschäftigt, wurde in Abschnitt 2.2.1 vorgestellt: Die TransitionForschung mit ihrem Konzept der MLP (Geels 2002, 2011). Darauf stützt sich auch der WBGU (2011, siehe Abschn. 2.2.2) in seiner Darstellung von Transformationsprozessen und möglichen Lösungswegen. Wie die MLP, so spricht auch der WBGU kleinen Nischenakteur*innen eine wichtige Rolle bei Transformationen zu, wobei der WBGU diese insbesondere zu Beginn des Prozesses sieht, wo es um die Verbreitung neuer Werthaltungen und das Experimentieren an neuen Innovationen geht, die als kleine Teilschritte einer Transformation dann von der Politik unterstützt und institutionalisiert werden müssen. Die MLP fokussiert dagegen 
meist auf die Prozesse der Diffusion in die Regime hinein (siehe Abschn. 2.2.1). Die Rolle der Politik wird über einzelne von dort agierende Nischenakteur*innen hinaus bei der MLP kaum betrachtet.

Vom WBGU (2011) wurde auch der Forschungsansatz der transformativen Forschung eingeführt, bei dem die Forschenden selbst Ideen entwickeln und verbreiten, um Transformationsprozesse anzustoßen und zu unterstützen. Der WBGU (2011) sowie andere zentrale Autor*innen, die sich mit der transformativen Forschung beschäftigen (u. a. Schneidewind und Augenstein 2016) setzen zwar auf der MLP sowie weiteren theoretischen Konzepten (u. a. Kristof 2010) auf, doch trotzdem bleiben bislang einige Fragen offen und der Ansatz erscheint weiterhin nicht hinreichend theoretisch fundiert. So bleibt unklar, ob die verschiedenen kleinen Innovationen und Maßnahmen dabei wirklich einzelne Schritte einer Transformation darstellen oder eher die entsprechenden Werthaltungen verbreiten, die als Grundlage notwendig sind. Wie diese Experimente gestaltet sein müssen, damit sie Transformationspotenzial haben und auch den geforderten Paradigmenwechsel herbeiführen können, wird ebenfalls nicht deutlich. Daneben ist bisher unklar, wie die verschiedenen beobachteten Transformationsblockaden überwunden werden können (WBGU 2011, S. 346).

Hier zeigen sich einige Überschneidungen mit der Theorie des diskursiven Institutionalismus, bei der es auch um die Entwicklung, Verbreitung und Umsetzung von neuen Ideen, sowohl für neue Instrumente als auch alternative Paradigmen, geht, die unterschiedliche Arten von Wandel - so auch Transformationen - mit sich bringen können. Wann dies laut diskursivem Institutionalismus erfolgreich gelingt, welche Voraussetzungen dafür vorliegen müssen und wie die Veränderungsprozesse ablaufen, wird im folgenden Kapitel erläutert. Am Ende dieses Kapitels (Abschn. 3.5) werden dann die Transformationsverständnisse der transformativen Forschung und der MLP mit der Transformationsdefinition des diskursiven Institutionalismus verglichen und Schlussfolgerungen für die Analyse gezogen. 
Open Access Dieses Kapitel wird unter der Creative Commons Namensnennung 4.0 International Lizenz (http://creativecommons.org/licenses/by/4.0/deed.de) veröffentlicht, welche die Nutzung, Vervielfältigung, Bearbeitung, Verbreitung und Wiedergabe in jeglichem Medium und Format erlaubt, sofern Sie den/die ursprünglichen Autor(en) und die Quelle ordnungsgemäß nennen, einen Link zur Creative Commons Lizenz beifügen und angeben, ob Änderungen vorgenommen wurden.

Die in diesem Kapitel enthaltenen Bilder und sonstiges Drittmaterial unterliegen ebenfalls der genannten Creative Commons Lizenz, sofern sich aus der Abbildungslegende nichts anderes ergibt. Sofern das betreffende Material nicht unter der genannten Creative Commons Lizenz steht und die betreffende Handlung nicht nach gesetzlichen Vorschriften erlaubt ist, ist für die oben aufgeführten Weiterverwendungen des Materials die Einwilligung des jeweiligen Rechteinhabers einzuholen.

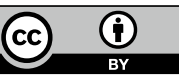

